Commentary on Integrating Cognitive-Behavioral and Cognitive-Interpersonal Case Formulations: A Case Study of a Chinese American Male

\title{
Being Culturally Sensitive is Not the Same as Being Culturally Competent
}

\section{WEI-CHIN HWANG ${ }^{\text {a,c }}$ \& JEFFREY J. WOOD ${ }^{\mathrm{b}}$}

\author{
${ }^{\text {a }}$ Department of Psychology, Claremont McKenna College \\ ${ }^{\mathrm{b}}$ Department of Education, University of California, Los Angeles \\ ${ }^{\mathrm{c}}$ Correspondence concerning this article should be addressed to Wei-Chin Hwang, Department of Psychology, \\ Claremont McKenna College, 850 Columbia Ave, Claremont, CA 91711 \\ Email: whwang@cmc.edu
}

\begin{abstract}
Empirically supported treatments have been found to be effective for psychiatric problems among Caucasian American clients. However, there continues to be little research conducted on the efficacy of such treatments when used with ethnic minority clients. Liu (2007) does an excellent job in using cognitive-behavioral and cognitive-interpersonal case formulations to develop an effective treatment plan for TC, a Chinese American male client. Moreover, this case study highlights the importance of cultural competency and cultural adaptation of empirically supported treatments when working with clients from diverse backgrounds. Herein, we summarize the literature on cultural competency and adaptation of therapeutic services, and discuss the cultural complexities involved with treating ethnic minorities.
\end{abstract}

Key words: cultural competence; adaptation; therapy; cognitive-behavioral; Chinese American; Chinese; ethnic minority

\section{INTRODUCTION}

In recent years, there has been an initiative to establish, define, and validate Empirically Supported Treatments (ESTs) in the U.S. (American Psychological Association Task Force on Psychological Intervention Guidelines, 1995; Task Force on Promotion and Dissemination of Psychological Procedures, 1995). However, ethnic minorities for the most part have been left behind in this initiative and the little research that is available demonstrates that ethnic minorities are less likely to receive quality health services and evidence worse treatment outcomes than White Americans (Institute of Medicine, 1999; U.S. Department of Health and Human Services, 2001). As the demographics of the U.S. change rapidly, this critical lacuna in our knowledge along with our under-preparedness to effectively treat ethnic minorities suffering from mental illnesses will become more apparent.

Currently, we do not know how to best address this dilemma. Numerous agencies, including the American Psychological Association (APA), the Surgeon General's office, and the Institute of Medicine have put out forth calls and guidelines for mental health providers to be 
Being Culturally Sensitive is Not the Same as Being Culturally Competent

W. Hwang \& J.J. Wood

Pragmatic Case Studies in Psychotherapy, http://pcsp.libraries.rutgers.edu

Volume 3, Module 3, Article 3, pp. 44-50, 07-27-07 [copyright by authors]

more effective in treating those from diverse backgrounds and offer guidelines for culturally competent practice (American Psychological Association, 2003; U.S. Department of Health and Human Services, 2001). Although these guidelines often help practitioners understand the importance of cultural competency, they are too general and leave professionals who want and need cultural competence with few skills that they can implement into actual practice.

Cultural competency is a very broad term that is hard to define and even more difficult to teach. The most widely accepted definition of cultural competency refers to having cultural selfawareness, knowledge, and skills that facilitate the delivery of effective services to ethnically and culturally diverse clientele (D.W. Sue, 1982; D.W. Sue, Arrendondo, \& McDavis, 1992). In describing the complexities of cultural competence, Lo \& Fung (2003) add that it is also important to distinguish between generic and specific cultural competencies, or the knowledge and skill sets needed in any cross-cultural encounter versus that which is necessary to work with a specific ethnocultural group.

A common but problematic assumption made by health care professionals is that learning about the client's culture will help them become more culturally effective. Unfortunately, this way of thinking only reinforces the problematic assumption that the difficulty of culture is located in the client as the other, rather than the cultural difference between the client and the provider. Hwang (2006) stresses the importance of an interactional perspective between the client and the provider, noting that cultural competence requires the therapist to understand his/her cultural self in relation to others, as well as a substantive understanding of the client.

Unfortunately, the required diversity training has yet to be instituted into undergraduate and graduate training programs. Even if instituted, taking such courses is only the beginning of one's journey to being more culturally competent and does not provide the requisite knowledge and skills necessary to be effective in treating diverse populations. Cultural issues need to be integrated into all aspects of psychology training programs in order to adequately prepare young professionals to work with our rapidly diversifying population. This is especially important because by 2050 over half of the U.S. will be non-European American (Day, 1996).

Despite the advances that have been made, there is still no uniform methodology or framework for adapting and modifying treatment interventions for ethnic minority groups, or for implementing such adaptations into widespread practice (Hwang, 2006). Many mental health practitioners already make their own personal modifications to therapy to better meet the needs of diverse clientele. However, these modifications may not be systematic or driven by a clear conceptual framework. Fortunately, there is hope that we can improve treatment outcomes for ethnic minorities. A recent meta-analytic study by Griner and Smith (2006) found that interventions targeted to a specific cultural group were four times more effective than interventions targeting multiple ethnic groups. Cultural adaptations in these 76 studies were loosely defined (e.g., providing therapy in the client's native language, addressing cultural values, consultation with individuals from the client's culture, being served at an ethnic-specific treatment center, cultural sensitivity training of staff, providing extra services such as child care and administration of written materials for illiterate clients, and specific cultural adaptations of a traditional mental health intervention). If more systematic and empirically guided therapy 
Being Culturally Sensitive is Not the Same as Being Culturally Competent

W. Hwang \& J.J. Wood

Pragmatic Case Studies in Psychotherapy, http://pcsp.libraries.rutgers.edu

Volume 3, Module 3, Article 3, pp. 44-50, 07-27-07 [copyright by authors]

adaptations and trainings of cultural competence are developed, even larger therapeutic effects would be expected.

\section{ANALYSIS OF THE CASE OF TC}

Overall, the case of TC (Liu, 2007) serves as an excellent example of how to culturally adapt empirically supported treatments for ethnic minority clients. The author offers a well thought out case conceptualization based on various cognitive therapies. In addition, she is wellread on the growing body of literature on providing care for Chinese and Chinese American clients, and as with many therapists, she makes her own cultural adaptations to therapy. Below, we offer our thoughts about the case.

\section{Understanding Cultural Complexities and the Avoidance of Stereotypes.}

Clinicians who treat clients from diverse backgrounds often encounter the problem of how to best understand the client and his/her problems. Specifically, how does one understand whether the client's behaviors and problems stem from individual traits or cultural characteristics? In the case of TC, Dr. Liu comes across this challenge when negotiating TC's self-defeating cognitions, which when interpreted from a Chinese cultural context, took on a qualitatively different meaning. Specifically, whereas in Western culture negative self-defeating thoughts are generally understood as a road block to reaching one's goals, in Chinese culture, such self and social criticism in some circumstances serve as motivators to achieve.

What function did they serve for TC? This question highlights the dynamic, temporal, and contextual nature of cultural strengths and individual dysfunction. As Dr. Liu notes, they initially served as a cultural strength and helped motive TC to achieve earlier in his life.

However, it seems that TC reached an impasse in his development and internalized these critical familial and individual self statements without the benefit of adult reflection on and mature adaptation of them. The goal here is not to judge culture or to label cultures as being better or worse. In fact, individuals from China have been living in good health for thousands of years, and Chinese cultural characteristics serve as a source of strength for its people. Instead, the goal is to understand how the individual client operates within a cultural context and to determine why he/she has now reached an unhealthy state. For example, healthy Chinese Americans may have both a positive understanding of self and use self-defeating thoughts to challenge themselves to work harder. These thoughts may in turn activate positive self-statements and help rally the client's self-efficacy, drive, and motivation. Moreover, in a healthy social environment, making negative self-statements may sometimes serve the social function of rallying social support and communicate that the social environment should no longer use critical statements to motivate the client because the client is already humble and doing it him/herself. In this situation, the nature of the social feedback loop is supposed to change from being critical to being more supportive.

For TC, the cycle became dysfunctional when the healthy chain of events fell astray: appropriate social feedback either failed or was overloaded with excessive expectations, and selfdeprecating thoughts began to impede his functioning. It is also equally important to remember 
Being Culturally Sensitive is Not the Same as Being Culturally Competent

W. Hwang \& J.J. Wood

Pragmatic Case Studies in Psychotherapy, http://pcsp.libraries.rutgers.edu

Volume 3, Module 3, Article 3, pp. 44-50, 07-27-07 [copyright by authors]

that not all Chinese people derive strength from critical self-statements and that many have very positive and supportive social and self supports. Thus, it may be inaccurate to apply broad stereotypes to individuals because these stereotypes are often more complex than their face value.

S. Sue (1998) aptly describes the Liu's challenge in working with TC. S. Sue cautioned that one danger associated with trying to be more culturally competent is the stereotyping of ethnic minorities and the use of general strategies when treating diverse clientele. Specifically, he warned that those trying to become more culturally competent may inadvertently take general recommendations to heart, apply what they have learned rigidly, and not consider the diversity of people within each cultural group. For example, a therapist who takes the recommendation that Chinese Americans are humble and that critical self-statements are detrimental to the client may be surprisingly mistaken when they try to get the client to use positive self-statements, which end up coming across as awkward and ineffective for that particular client. It may be that this particular client needs to understand why their critical self statements have failed to serve their function, and to help them use these critical statements to activate positive, motivating behaviors. Dynamic sizing (S. Sue, 1998), or the skill of knowing when to generalize and when to flexibly individualize treatments based on the client's characteristics, is an important skill to learn if practitioners are to prevent rigid overgeneralizations and increase their cultural competence.

Similar to dynamic sizing, a common issue that comes up in cultural competency training, supervision, and culture and mental health courses is the clinician's desire to separate out the clinical from the cultural. This is a natural question to ask because clinicians need to make a clinical assessment, understand the etiology of the problem, and diagnose the client. However, it is an awkward question to apply because clients do not have distinctly isolated clinical and cultural aspects of self. For example, the series of social and personal events that led up to TC's depression could be interpreted as a clinically dysfunctional and stressful environment. These events could also be interpreted as being culturally incongruent. However, in the end, the series of events that led up to and were maintaining TC's depression are best described as clinical in a culturally Chinese way. Similarly, some people may feel that being poor is the same in all cultures and the best method to address poverty should be a general economic one. However, this strategy would not address the issue of how Chinese Americans who are poor are poor in a specifically Chinese American way, and that being poor in this culturally specific way must be addressed directly for clinical gains to be made.

\section{Cultural Adaptations Made in Treatment}

The case of TC highlights the multiple ways in which culture can influence mental health. For example, cultural differences in communication (e.g., TC having less eye contact with the therapist) could influence diagnostic accuracy if not interpreted through a clinicalcultural lens (e.g., he was anxious, and since people from Chinese cultural backgrounds stereotypically have less eye contact with authority figures, TC's strikingly poor eye contact could have been a function of both a cultural tendency exacerbated by his personal anxiety.) Differences in how perceived stigmatizing from seeking help from a mental health practitioner can also influence (a) whether the client comes back and (b) the degree to which the therapist 
Being Culturally Sensitive is Not the Same as Being Culturally Competent

W. Hwang \& J.J. Wood

Pragmatic Case Studies in Psychotherapy, http://pcsp.libraries.rutgers.edu

Volume 3, Module 3, Article 3, pp. 44-50, 07-27-07 [copyright by authors]

needs to orient the client to therapy to prevent him/her from dropping out, both of which Dr. Liu addressed in treatment. Making an extra effort to normalize the client's experiences also helped to make the therapeutic experience more positive and to strengthen the client-therapist working alliance. Cultural perceptions that hard work rather than personal ability is the most important determinant of achievement could also be used to motivate the client to complete homework assignments and to help him/her become more optimistic about the beneficial effects of therapy.

In the case of TC, Liu highlights how many of the adaptations she made to treatment map onto the 18 principles of adapting cognitive-behavior therapy for Chinese Americans developed by Hwang et al. (2006). Hwang (2006) expanded on these 18 principles of adaptation by developing a systematic framework for therapy adaptation. The Psychotherapy Adaptation and Modification Framework (PAMF) uses a framework consisting of 6 therapeutic domains, 25 therapeutic principles, and corresponding rationales to guide therapeutic cultural adaptations. The domains include: (a) dynamic issues and cultural complexities, (b) orienting clients to psychotherapy and increasing mental health awareness, (c) understanding cultural beliefs about mental illness, its causes, and what constitutes appropriate treatment, (d) improving the clienttherapist relationship, (e) understanding cultural differences in the expression and communication of distress, and (f) addressing cultural issues specific to the population. Broader domains identify general areas that practitioners should think about when modifying their approach for treating their clients. More specific therapeutic principles provide detailed instruction on the types of adaptations that may be important to make for a particular group. Corresponding rationales help the practitioner understand why some of these modifications should be made and how they might be beneficial. This three-tiered approach to presenting cultural adaptations to therapy was developed in order to make the PAMF more accessible, userfriendly, and adaptable for use with other diverse populations.

Dr. Liu employed many of these therapy adaptations in treating TC. For example, she made a concerted effort to learn about TC's cultural beliefs and family background. She set up concrete observable goals and used identifiable indicators of improvement. She used many cultural bridges (e.g., chengyu, or Chinese metaphorical sayings) to help TC understand therapeutic concepts. She helped the client deal with feelings of shame and guilt associated with receiving mental health services by normalizing his experiences through psychoeducation and empathy. She also interpreted the client's cognitive, emotional, and somatic complaints through a clinical-cultural lens so that she could better address the different problems the client was experiencing.

One issue that was not addressed by Dr. Liu is how adapting therapeutic techniques might be approached by therapists who do not share the client's cultural background and first language. Many ethnic minority clients in the U.S. continue to be treated by individuals from different cultural groups - particularly European Americans. If therapy is conducted in a second language (for the client), do additional issues in cultural adaptation arise? Our experience suggests that the discomfort and awkwardness that naturally arises in conversations in which feelings and ideas cannot be fluently expressed in a second language (i.e., English) can quickly erode the therapeutic bond if specific consideration of this challenge and corresponding accommodations are not made. 
A very pragmatic first step is to acknowledge linguistic limitations and for the therapist to apologize for not being able to communicate in the client's native language (this is consistent with the self-deprecation expected in some Chinese cultural subgroups). Subsequent steps involve emphasizing the therapist's authentic desire to help in spite of these communication challenges, and engaging in brainstorming with the client to develop a range of options for overcoming the barriers - for example, bringing in colleagues, family or friends as translators for critical topics, using dictionaries or pictures, and speaking slowly and clearly enough to ensure that meaningful communication is occurring. It is important to note that speaking louder doesn't help a person understand a language and that it can sometime lead the client to feel like the therapist is yelling at them or that the therapist thinks that they are unintelligent. Gentle encouragement for the client to express themselves and patience on the therapist's part is essential to helping the client feel more comfortable.

In addition, there are other cultural issues that could come into play. For example, in some cases, therapist acknowledgement of the client's cultural tendencies towards politeness and respect for authority in a doctor-patient relationship that might promote passivity in therapy could be countered by acculturating the client to the culture of therapy and by emphasizing the importance of the client's being active. Repeating this point periodically and affirming clients for expressing themselves and for correcting the therapist can help clients overcome their reluctance and take a more participatory role therapy. This cooperative process also helps facilitate accurate therapist understanding of the case. In addition, clinicians who do not share the same cultural background of their client must acknowledge their own limitations and seek consultation. They should also be wary of their own feelings of discomfort when working with clients from backgrounds with which they are not familiar. They should also be aware of their own ethnocultural counter-transference and not overcompensate by acting overconfident when feelings of anxiety arise, or under-compensate by behaving in an ineffectual and nonauthoritative manner.

In sum, Dr. Liu made a number of effective cultural adaptations to the case conceptualization and therapeutic treatments. These adaptations helped the therapist build a positive working relationship with the client and made the integrative cognitive-behavioral and cognitive-interpersonal treatment more effective. Her treatment strategy was practical, consumer-driven, and used an integrative, clinical-cultural lens of understanding. This case helps highlight the cultural complexities involved in treating people from diverse backgrounds. It also helps practitioners make the shift from cultural awareness to clinical application. Liu demonstrates how being culturally sensitive and aware is different from being culturally competent per se. 


\section{REFERENCES}

American Psychological Association Task Force on Psychological Intervention Guidelines. (1995). Template for developing guidelines: Interventions for mental disorders and psychological aspects of physical disorders. Washington, DC: American Psychological Association.

American Psychological Association (2003). Guidelines on multicultural education, training, research, practice and organizational change for psychologists. American Psychologist, 58, 377-402.

Day, J. C. (1996). Population projections of the United States by age, sex, race, and Hispanic origin: 1995 to 2050, U.S. Bureau of the Census, Current Population Reports, pp. 25-1130. Washington, DC: U.S. Government Printing Office.

Griner, D., \& Smith, T. B. (2006). Culturally adapted mental health interventions: A metaanalytic review. Psychotherapy: Theory, research, practice, training, 43, 531-548.

Hwang, W. (2006). The Psychotherapy Adaptation and Modification Framework (PAMF): Application to Asian Americans. American Psychologist, 61, 702-715.

Hwang, W., Lin, K., Cheung, F., \& Wood, J. J. (2006). Cognitive-behavioral therapy with Chinese Americans: Research, theory, and clinical practice. Cognitive and Behavioral Practice, 13, 293-303.

Institute of Medicine (1999). Unequal treatment: Confronting Racial and Ethnic Disparities in Health Care. U.S.: National Academy of Sciences Press.

Liu, E.T. (2007). Integrating cognitive-behavioral and cognitive-interpersonal case formulations: A case study of a Chinese American male. Pragmatic Case Studies in Psychotherapy [Online], Vol. 3(3), Article 1, pp. 1-33. Available: http://hdl.rutgers.edu/1782.1/pcsp_journal.

Lo, H., \& Fung, K. P. (2003). Culturally competent psychotherapy. Canadian Journal of Psychiatry, 48, 161-170.

Sue, D.W. (1982). Position paper: Cross-cultural counseling competencies. Counseling Psychologist, 10, 45-52.

Sue, D.W., Arredondo, P., \& McDavis, R. J. (1992). Multicultural counseling competencies and standards: A call to the profession. Journal of Multicultural Counseling \& Development, 20, 64-88.

Sue, S. (1998). In search of cultural competence in psychotherapy and counseling. American Psychologist, 53, 440-448.

Task Force on Promotion and Dissemination of Psychological Procedures. (1995). Training in and dissemination of empirically-validated psychological treatments: Report and recommendations. Clinical Psychologist, 48, 3-23.

U.S. Department of Health and Human Services. (2001). Mental Health: Culture, Race, and Ethnicity - A Supplement to Mental Health: A Report of the Surgeon General. Rockville, MD: U.S. Department of Health and Human Services, Public Health Service, Office of the Surgeon General. 